\title{
For a childhood story. Social representations, childhood memories and educational abuses
}

\section{Per una storia dell'infanzia. Rappresentazioni sociali, memorie bambine e abusi educativi}

\section{Francesca Borruso}

\begin{abstract}
The essay deals with the issue of violence against children between the Nineteenth and Twentieth centuries, by trying to intertwine - through a variety of historical sources - macro history with micro history, history of ideas with real educational practices, history of ruling classes with that of subordinate classes. The topic of "discovering the sense of childhood" - understood as awareness of the child life specificity that has become a "value" in the Western World - eventually seemed to have put into crisis the old way of thinking about childhood and education. However, this thorough renewal of educational thinking does not prevent the old interpretive paradigms of childhood - viewed as a season of defective life that should be corrected and suppressed - to survive in some scientific areas. From life histories violent educational practices emerged, making extensive use of corporal punishment and sophisticated psychological conditioning techniques. From these histories of real childhood it seems to emerge the gap - that always existed at all times - between ideas and real educational practices.
\end{abstract}

Keywords: childhood, violence, education, education to feelings, life histories, mentality.

\section{L'infanzia nella ricerca storiografica}

Secondo lo storico Lloyd deMause, la storia dell'infanzia nella cultura occidentale sarebbe stata attraversata da forme così efferate di violenza, da poter essere definita un incubo, dal quale avremmo iniziato ad affrancarci solo negli ultimi anni del secolo scorso. Si sarebbe così passati da pratiche di violenza e di rifiuto tipiche dei secoli passati - l'infanticidio, l'abuso sessuale, le punizioni corporali, l'abbandono - a forme di aiuto, cura e valorizzazione dell'infanzia solo a partire dall'età contemporanea (deMause, 1994). Una interpretazione condivisibile sotto molti aspetti, contestabile solo per una eccessiva linearità delle periodizzazioni storiche da lui individuate, scandite in almeno sei tappe, che l'Autore ritiene si succedano evolutivamente, in relazione 
alla qualità del rapporto genitori-figli, verso forme di superamento della violenza la quale, purtroppo, sappiamo essere presente ancora oggi nelle società attuali, anche nei contesti più insospettabili (Cambi, Ulivieri, 1990; Riva, 1993; Ulivieri, 2014). Nel 2015, infatti, si stima che siano oltre 91mila i minorenni maltrattati seguiti dai Servizi Sociali nel nostro Paese secondo la prima Indagine nazionale sul maltrattamento dei bambini e degli adolescenti in Italia condotta dall'associazione «Terre des Hommes» e dal Cismai. Dati sconvolgenti e che ribadiscono la necessità di una riflessione radicale sull'educazione ai sentimenti, agli affetti e alle emozioni nelle società occidentali del benessere, massmediatiche, interculturali, anomiche e postmoderne da parte di tutte le scienze dell'educazione (Borruso, Cantatore, Covato, 2014).

La storia della violenza nei confronti dell'infanzia, però, deve intrecciarsi con quella che è stata ritenuta dalla storiografia contemporanea una delle più grandi rivoluzioni dell'età moderna, ossia il progressivo riconoscimento della condizione infantile intesa come valore in Occidente, avvenuto a partire dal XVI secolo in poi. Philippe Ariès, uno dei più noti e controversi Autori di questa tesi storiografica, l'ha identificata con il sorgere di un sentimento dell'infanzia che, dalla modernità in poi, si sarebbe lentamente evoluto contestualmente al delinearsi del «sentimento della famiglia», sempre più considerata come il luogo primario della vita affettiva ed educativa per le nuove generazioni. Una valorizzazione nuova e inedita dell'infanzia rispetto al passato, da intendersi come consapevolezza della specificità della vita infantile, colta nella sua diversità dalla vita adulta, idealizzata per alcuni aspetti della sua natura (la debolezza, la tenerezza) e resa destinataria di cure materiali ed educative appositamente concepite (Ariès, 1973). Un nuovo sentire, che già nel Settecento ha uno dei suoi massimi interpreti in Jean-Jacques Rousseau il quale, nella sua celebre opera L'Emilio (1762), idealizzando e accostando l'infanzia al mito del «buon selvaggio» incorrotto e moralmente retto, ipotizzava il ritorno a un ipotetico stato di natura - almeno sul piano educativo - per rifondare quell'uomo nuovo che avrebbe portato il germe del rinnovamento etico-morale e sociale nella società al fine di dar vita a nuove forme di convivenza umana e sociale (Boas, 1966; Todorov, 2002). La critica radicale di Rousseau contro le pratiche educative del tempo e contro l'idea di infanzia dei suoi contemporanei, ebbe una vasta eco nelle classi elevate e colte del tempo e certamente contribuì ad orientare questa nuova concezione dell'infanzia, che raggiunse la sua massima espressione culturale proprio nella società europea borghese e capitalistica (Trisciuzzi, Cambi, 1989). Infatti, nonostante le tante critiche rivolte all'interpretazione di Ariès per la collocazione cronologica del fenomeno, per le fonti utilizzate e per la loro interpretazione, ma soprattutto per l'interpretazione evoluzionistica del sentimento dell'infanzia (deMause, 1994; Becchi, 1979; Pollock, 1983) - sembra 
unanimemente condiviso il fatto che questa nuova idea d'infanzia troverà la sua maggiore espressione di rinnovamento pedagogico proprio fra Otto e Novecento. Così, se nel corso dell'Ottocento, sul piano ideale e pedagogico, si cerca di mitigare la tradizionale concezione negativa dell'infanzia, intesa come stagione della vita caratterizzato da mancanze, limiti, minorità, tra la fine del secolo e l'inizio del Novecento entra definitivamente in crisi il vecchio modo di concepire l'infanzia e l'educazione, grazie allo sviluppo dell'attivismo, della psicoanalisi, della psicologia dello sviluppo e della moderna pedagogia scientifica e sperimentale (Pironi, 2010). Una educazione rinnovata che nel pensiero di John Dewey, ad esempio, si declinerà anche come un progetto di rinnovamento democratico della società, identificando così il rispetto per l'infanzia con il culto del pieno e armonico sviluppo dell'intera umanità.

Ma se sul piano della storia delle idee evolve la concezione dell'infanzia, non necessariamente in quella direzione e nel medesimo tempo storico mutano sia le pratiche educative delle diverse classi sociali (per quanto l'infanzia borghese e aristocratica sia la prima a godere di questi cambiamenti), sia l'immaginario collettivo di una società (Covato, 2014). Dal punto di vista storiografico, infatti, dobbiamo distinguere fra un'idea di infanzia alla cui elaborazione contribuiscono la storia delle idee e la trattatistica pedagogica così come i pregiudizi, le paure e l'inconscio collettivo di una società, in un rapporto di circolarità e contaminazione reciproca in cui è difficile districarne le interferenze reciproche - le idee sono sempre attraversate dall'immaginario di una società - (Vovelle, 1982) e le condizioni di vita reali, spesso in stridente contrasto con le teorie pedagogiche. Le condizioni di vita di tante bambine e tanti bambini, per quanto con fenomenologie diverse nelle diverse classi sociali, fino al Novecento inoltrato erano caratterizzate da non irrilevanti pratiche di violenza, di abusi educativi, di sfruttamento, così come da un rapporto di severa sottomissione alla potestà e allo ius corrigendi dei genitori. Prassi educative diverse, infatti, caratterizzavano l'infanzia borghese o aristocratica da quella contadina o operaia, così come un'infanzia vissuta in città, nel pieno dell'urbanesimo industriale, era diversa da una vissuta in campagna, o ancora quella di una bambina da quella di un bambino (Becchi, 1996; Covato, Ulivieri, 2010). Si tratta di una antinomicità complessa, quindi, che non riguardava solo la dialettica fra la teoria e la prassi e che, soprattutto nel corso del XXesimo secolo, sembrava emergere con ancora più fragore, poiché nuove teorie e nuove sensibilità pedagogiche si scontravano con le pratiche educative reali.

Proveremo, così, ad intercettare fra Otto e Novecento, soprattutto nel pensiero scientifico dell'Italia borghese post unitaria, un'idea di infanzia che anche se emancipata per certi aspetti, inglobava in sé un paradigma interpretativo di lunga durata di un'immagine d'infanzia svalutata, da correggere e reprimere (Cambi, Ulivieri, 1988). Su alcune storie di vita, infine, 
rifletteremo per comprendere lo scarto, sempre esistente in tutti i periodi storici, fra le idee e le prassi educative reali, cercando di intrecciare macro e micro storia, la storia delle idee con le prassi educative reali, la storia delle classi dominanti e di quelle subalterne, così come la molteplicità ed eterogeneità delle fonti storiche in gioco, tali loro malgrado, sempre più complesse, inedite ed estese (Braudel, 1986).

\section{Educare un piccolo essere perverso e dispettoso}

La medesima cultura scientifica positivista che fra Otto e Novecento aveva valorizzato l'infanzia (Maria Montessori, Giuseppe Montesano, Sante De Sanctis), paradossalmente sembra costituire, per altri versi, il medesimo sfondo culturale di un altro filone di studi, soprattutto di matrice antropologicoeducativa, in cui emerge un'idea di infanzia condizionata da arcaici e poco camuffati pregiudizi (Guarnieri, 2006).

Cesare Lombroso, ad esempio, professore di psichiatria e di antropologia criminale all'Università di Torino, e che gode di indubbia fama internazionale, analizzando le tendenze a delinquere del minore, ritiene che ogni bambino possa ben definirsi «collerico, egoista, crudele, vendicativo, geloso, bugiardo, ladro, privo di sentimenti affettivi, pigro, imprevidente, vanitoso, osceno» (Lombroso, 1883, p. 10). In ciascun bambino, insomma, sono presenti i germi della pazzia morale e del delitto, poiché, proprio come la donna, l'infanzia viene ritenuta una tappa evolutivamente inferiore dello sviluppo. L'inferiorità e la debolezza del bambino, inoltre, alimentano il suo ragionamento astuto, ritenuto una forza istintiva tipica di tutti gli esseri inferiori. Da qui il pianto facile, la risposta pronta, la menzogna audace del bambino che in tal modo trae in inganno l'adulto, trasformandosi, in tal caso, nel più forte della relazione, potendo rendere vittime della sua astuzia gli adulti. Una interpretazione che il suo Autore ritiene scientifica perché fondata sull'osservazione diretta dei ragazzi della scuola italiana, sottoposti ad un sistema di misurazione antropometrico complesso, da cui lo scienziato deduceva tendenze comportamentali e strutture di personalità. "Quando in chi e perché e come cessa in essi quella irascibilità affettiva, quella mancanza di senso morale che ne fa veri delinquenti nati?» scriveva Lombroso nel 1887 in una Prefazione ad un volume di Perez (Lombroso, 1887). Una tendenza antisociale che Lombroso ritiene connaturata ad un orientamento biologico, mentre altre ricerche sul campo coeve, spiegavano già allora con ragioni di carattere sociale: le famiglie, l'ambiente di vita, la strada con le sue malefiche suggestioni erano le ragioni portate avanti da alcuni esponenti della psichiatria italiana (Vidoni, 1924, p. 24). 
Lo stesso antropologo Paolo Mantegazza, audace contestatore del pensiero lombrosiano, nei suoi studi sulla fisiologia dei sentimenti asseriva che nel «fanciullo è istintiva altresì la passione di far dispetti, di tormentare $\mathrm{i}$ compagni, d'inventar giuochi, scherzi che sian molesti agli altri» (Mantegazza, 1889, p. 25). Tale passione che si manifestava già dall'infanzia, poteva durare tutta la vita se una saggia educazione unita ad una forte volontà non fossero riuscite a contenerla. Una visione dell'infanzia, quindi, che riteneva pericolose e da contrastare delle tendenze connaturate alla natura umana, biologicamente o socialmente determinate - su questo gli studiosi si contendevano le diverse interpretazioni - a cui l'educazione doveva porre rimedio.

Un filone interpretativo che traspare anche nel Dizionario illustrato pedagogico, pubblicato tra il 1892 e il 1903 e diretto da Luigi Credaro e Antonio Martinazzoli, dove nella voce dedicata allo psicologo e pedagogista Bernard Perez, veniva sostenuta la tesi che educatori, medici e genitori dovessero prestare attenzione a che le naturali tendenze non si prolungassero oltre l'età prevista: «Come avviene quel disordine, quel tumulto di sensazioni sfuggenti ed opposte per cui il fanciullo sembra un matto? Fino a quel punto si può considerare normale un tale stato? Non vi ha nulla di morboso, in quella eccessiva instabilità di impulsi? Non ha bisogno di correttivi e di deviazioni?» (Gelmini, 1892, p. 229).

L'educazione, in questa prospettiva, aveva la funzione di integrare il fanciullo all'interno delle regole dominanti, contenendolo, reprimendolo, correggendolo. Una visione dell'infanzia che, se da un canto si collegava ad un paradigma di lunga durata - vedi l'idea, già risalente a Cartesio, di un'infanzia vista come una malattia da superare al più presto - d'altro canto, in quel periodo storico metteva in crisi quel paradigma interpretativo di roussoiana memoria esaltato nel romanticismo, che vedeva l'infanzia come la stagione della bontà. Una visione angelicata dell'infanzia, che di lì a poco, nei primi anni del Novecento, sarebbe stata ulteriormente messa in crisi dalle dirompenti scoperte freudiane che disvelando la sessualità del bambino, il suo essere infinitamente desiderante, lo avrebbero identificato come un piccolo perverso polimorfo votato alla continua ricerca del piacere attraverso una pluralità di zone erogene (Freud, 2003). Cosicché, ci sembra possibile poter affermare che nell'ambito di questa idea d'infanzia della scienza positivista di fine Ottocento si mescolassero vecchie credenze e nuove sensibilità, forse anche confusamente intrecciate, supportate però dall'ambizione di un pensiero scientifico che voleva affrancarsi dai pregiudizi irrazionali del passato per fondarsi, invece, su quelli che la scienza del tempo, riteneva riscontri oggettivi e predittivi: esami antropometrici, test mentali, reazioni fisiologiche, stimoli indotti in laboratorio.

Non vogliamo affatto liquidare negativamente il positivismo italiano che invece, come scrive Patrizia Guarnieri, tra le sue oscurità, pregiudizi e 
ideologie - a tratti maschilisti, adultocentrici, moralisti o razzisti - ha avuto diversi meriti, forse ancora poco valorizzati. Fra i tanti - nel tentativo di costruire una norma interpretativa che distinguesse la normalità dall'anormalità anche nel mondo infantile, secondo quel meccanismo binario oppositivo e di esclusione e che Foucault ha disvelato essere presente nelle moderne società disciplinari (Foucault, 1971) - «la specificità delle differenze, [...] fu forse l'originale e assai sofferta scoperta della cultura positivista» (Guarnieri, 2006, p. 272). Una interpretazione negativa dell'infanzia che non implicava né l'idea di peccato né quella di colpa, poiché si trattava di un "naturale" comportamento cattivo, dispettoso, ribelle, disordinato. Una naturalità del comportamento perverso, quindi, che non poteva che esaltare il ruolo dell'educazione, richiamata alla centralità del suo compito. Solo un'educazione adeguata avrebbe potuto porre rimedio alla follia o delinquenza incipiente del bambino, ritenuto normalmente cattivo, modificando i suoi istinti, reprimendoli, correggendoli in vista di una integrazione sociale del bambino e poi dell'adulto, rispettosa della norma e della morale dominante. Un paradigma educativo coercitivo e autoritario, destinato al bambino privatizzato del mondo borghese, intrappolato in un progetto educativo di sottomissione, dominio e governo sempre più capillari in cui l'infanzia era vista come stagione della vita perversa e anarchica, scandalosamente erotica e pulsionale, profondamente libera e spontanea (Becchi, 1979).

\section{Con la dolcezza delle pene}

La concezione dell'educazione intesa come conformazione ad un modello dominante impositivo e conformativo, che sottende un'idea di inferiorità dell'infanzia, è la più conosciuta sul piano storico e si è declinata in forme di controllo e di condizionamento precoce e violento, facendo ampiamente uso di punizioni corporali e di sofisticate tecniche di condizionamento psicologico. Un'educazione fondata sul sadismo e la prevaricazione, una pedagogia nera secondo la psicoanalista svizzera Alice Miller, presente in tutte le formazioni sociali e in tutti i tempi storici perché insita nell'educazione tout court secondo l'Autrice e che, sul piano psicologico, si spiega con il meccanismo della coazione a ripetere, non solo perché l'adulto ripropone il già subito, ma soprattutto perché si illude di poter dominare le proprie paure trasmettendole alla generazione successiva (Miller, 1987, p. 5).

Dalla trattatistica pedagogica settecentesca emerge, infatti, un filone di pensiero che prospettando un'educazione violenta sia sul piano fisico sia sul piano psicologico, mira ad ottenere un'assoluta obbedienza all'autorità fin dalla più tenera età. Eliminare l'ostinazione dai loro teneri animi, privarli della loro 
volontà, batterli non solo per i loro errori ma anche per la loro testardaggine, minacciarli preventivamente per orientare la loro condotta, incutere loro paura, evidenziare le loro fragilità e i loro limiti, umiliarli e deriderli pubblicamente anche davanti ad estranei, mortificarli per le loro debolezze, punirli a distanza di tempo per non essere accecati dall'ira e convincerli, così, della giustezza della punizione subita, sono alcuni dei tanti consigli pedagogici in cui è possibile imbattersi (Krüger (1752), Sulzer (1732) citati in Miller, 1987, p. 16). Esercizio della violenza, interiorizzazione della paura, squalifica e derisione sembrano essere i principali metodi utilizzati, all'interno di un processo educativo considerato come processo di condizionamento alle regole del mondo adulto, in cui ogni bisogno del bambino è negato. Un raffinato sadismo pedagogico che doveva essere realizzato soprattutto durante l'infanzia, poiché dopo, qualunque intervento educativo sarebbe stato inutile.

Fra Sette e Ottocento, il diffusissimo metodo delle punizioni corporali, che prima Erasmo da Rotterdam, Locke e poi Rousseau avevano condannato esplicitamente anche se con poco successo - visto che ancora nella prima metà del Novecento ne è documentato un ampio uso nelle classi borghesi come nelle classi lavoratrici oltre che nella vita scolastica - viene sostituito dalla sottile punizione psicologica, ravvisabile nelle forme del ricatto affettivo, dell'umiliazione, della colpevolizzazione, della sottrazione dell'amore, della minaccia, dei silenzi, dell'indifferenza affettiva. Si passa, insomma, dalla brutalità del supplizio alla dolcezza delle pene senza per questo superare la violenza insita nei dispositivi pedagogici dominanti (Foucault, 1975).

Dalle storie di vita reali cercheremo di far emergere questo complesso dispositivo pedagogico, nonostante le difficoltà insite sia nell'interpretazione di una memoria individuale che fa i conti con il diritto all'oblio e alle distorsioni narrative, sia nella decodifica degli impliciti, dei silenzi e dei non detti - che possono essere un altro volto della violenza - agiti nelle relazioni educative (Covato, 2014).

Ingmar Bergman, noto regista cinematografico svedese, nella sua autobiografia ci offre uno spaccato della sua infanzia nella evolutissima Svezia del primo Novecento, dove il padre un colto ma rigido pastore protestante alleva i suoi figli esercitando ancora un arcaico ius corrigendi paterno. Fra le punizioni, accanto alle busse tradizionali con la frusta, c'era la reclusione al buio nel guardaroba - terrorizzante ancora nel ricordo del figlio adulto - il digiuno, il divieto di uscire, la consegna in camera, colpi di verga sulle mani, compiti di matematica supplementari, tirate di capelli, la semplice emarginazione dalla vita familiare a tempo indeterminato. C'erano poi umilianti pratiche volte a colpevolizzare la debolezza del bambino, come quella di indossare una gonnellina rossa qualora il bambino si fosse urinato addosso $\mathrm{o}$ a letto, mentre $\mathrm{i}$ delitti più gravi richiedevano un vero e proprio processo 
domestico che si svolgeva prima al cospetto della madre e delle domestiche, poi davanti al padre:

Poi uno doveva dire quanti colpi di battipanni riteneva di meritare. Quando la punizione era stata decisa si prendeva un cuscino verde dall'imbottitura dura, venivano calati pantaloni e mutande e il criminale doveva sdraiarsi a pancia in giù sul cuscino, qualcuno lo teneva saldamente per il collo e i colpi venivano inferti. Non posso dire che facesse particolarmente male, dolorosi erano il rituale e l'umiliazione (Bergman, 1997, pp. 13-14).

Fra le forme di violenza psicologica non possiamo non annoverare la pratica delle minacce, spesso più penetranti della violenza fisica perché, come è noto, l'indeterminatezza della punizione ingigantisce la paura e il senso di colpa, annientando la vittima in un dolore che si perpetua nel tempo, che non conosce una fine. Scrive Franz Kafka in una celebre lettera al padre pubblicata postuma e mai recapitata, del 1920 circa, di non essere mai stato picchiato dal padre:

ma le tue urla, il volto paonazzo, le bretelle staccate in fretta e appoggiate sullo schienale della sedia per me erano ancora peggio. E come quando qualcuno sta per essere impiccato. Se lo impiccano davvero, allora muore e tutto finisce. Se invece deve partecipare a tutti i preparativi dell'impiccagione e solo quando il cappio gli penzola davanti alla faccia apprende di essere stato graziato, forse ne soffrirà per tutta la vita» (Kafka, 2011, p. 21).

Poi c'è l'indifferenza emotiva aggravata dall'emergenza della guerra, che annulla qualunque esigenza bambina. Silvia Vegetti Finzi in un recentissimo libro di memorie in chiave psicoanalitica (2015) riesce a dare voce ai suoi ricordi d'infanzia evidenziando, sia il procedere della mente bambina (paure, emozioni, riflessioni, strategie di sopravvivenza), sia la sua capacità di riscatto e di riprogettazione esistenziale nonostante il dolore, ripartendo proprio dal dolore. La presa di coscienza autobiografica e retrospettiva, come sappiamo, può avere in sé una profonda valenza educativa, sia perché si tratta di una comprensione sempre più profonda di sé, condivisibile e partecipabile, sia perché contiene i germi del cambiamento e del rinnovamento (Cavarero, 2001). E i bambini, sostiene l'Autrice - lasciandoci un vero e proprio messaggio di speranza nella vita - nonostante i tentativi di vero e proprio sabotaggio esistenziale che possono subire dagli adulti significativi, spesso inconsapevoli del proprio agire educativo, possono avere una grande capacità di resilienza, ossia di elaborazione e di superamento del trauma con le risorse interiori di cui dispongono (Vegetti Finzi, 2015, p. 74). Lasciata prima alla balia poi a degli zii per diversi anni, perché i genitori sono stati costretti a fuggire in Abissinia con il primogenito a causa delle persecuzioni razziali, i ricordi d'infanzia 
della Vegetti Finzi evidenziano il dolore inespresso per l'assenza degli adulti significativi, il vissuto infantile connesso al mancato riconoscimento, all'indifferenza degli adulti per lo più capaci di un amore distratto ed egoista, all'incomunicabilità che rende il mondo dei piccoli e quello dei grandi a tratti incommensurabile.

Infanzie borghesi irretite in questo doppio registro: da un canto sono curate perché nuovo e fenomenologicamente più evidente è l'investimento affettivo che i genitori sviluppano verso i figli, rendendoli oggetto di attenzioni sempre più sofisticate connotate dalla propria appartenenza di classe - l'abbigliamento, i giochi, le letture, il tempo libero, l'alimentazione, le proibizioni sessuali d'altro canto sono sottoposte a forme di indifferenza emotiva, così come ad un sofisticato corredo punitivo e di controllo (Cambi, Ulivieri, 1990). Il dominio paterno, inoltre, già attaccato nel secolo dei Lumi dagli intellettuali del tempo (come, ad esempio, Rousseau, Verri e Beccaria) ma la cui crisi profonda si sarebbe evidenziata soprattutto nel corso del Novecento (Zoja, 2000), nella cultura borghese ottocentesca occupa ancora una centralità rilevante: il padre governa con le sue leggi sulla famiglia, mentre la madre, sempre più confinata nel privato perché resa angelo del focolare, viene contenuta in una gabbia simbolica che si rivelerà più coercitiva del passato (Covato, 2007; 2014).

Diversa appare l'infanzia reale vissuta dai figli della classe operaia, contadina e proletaria, resa ancora più drammatica dalla povertà materiale $\mathrm{e}$ dai processi di urbanizzazione e di industrializzazione selvaggia che, fra Otto e Novecento, causeranno il fenomeno dei bimbi impiegati non solo nel lavoro dei campi, ma anche in fabbrica o nelle miniere (Revelli, 1977). Si deve proprio a un giovane Friedrich Engels una delle prime inchieste sulla condizione della classe operaia in Inghilterra (1892), in cui denuncia le durissime condizioni di vita e di lavoro dei bambini nelle vetrerie inglesi (Engels, 1972, pp. 236-238). In queste famiglie, afflitte dalla miseria, i bambini non sono certo oggetto di cure amorose ma di dominio e di sfruttamento disumano. Spesso sono le stesse famiglie che decidono di vendere i propri figli per liberarsi di una bocca in più da sfamare - secondo un'antichissima pratica di cui le fiabe mutuate dalla tradizione orale danno ampia testimonianza (Filograsso, 2012) - così come, soprattutto in alcune aree della penisola come l'Appennino parmense e alcuni comuni del sud e del centro Italia, orfani e trovatelli sono oggetto di un vero e proprio traffico finalizzato alla riduzione in schiavitù per svolgere $i$ più diversi mestieri girovaghi, oppure lavorare nelle fabbriche o per essere destinati all'accattonaggio o in attività criminali in paesi lontani. Una vera e propria tratta dei bambini bianchi, come la chiamavano i libri e i quotidiani dell'epoca che ne denunciavano l'esistenza (Luatti, 2016, p. 36). Sono tanti i romanzi, i racconti e le letture scolastiche destinate all'infanzia che hanno come protagonisti queste piccole figure di bimbi: spazzacamini, vetrai, suonatori 
ambulanti d'organetto, lustrascarpe, venditori di statuine di gesso, cerinai, fanciulli nelle miniere, nelle fabbriche, nei negozi, nei campi. Dalle avventure di David Copperfield di Charles Dickens, a quelle di Remi protagonista del romanzo Senza famiglia di Hector Malot, fino al personaggio di Rosso Malpelo nel racconto di Giovanni Verga, la letteratura ottocentesca europea è composta da un ricco e variegato panorama di bambini poveri, sfruttati, abbandonati, venduti (Cantatore, 2015).

Ma ancora negli anni Cinquanta del Novecento i bambini possono essere allontanati dalla propria casa d'origine e dati in affitto per brevi periodi ad altre famiglie per svolgere attività criminali, come emerge da un'inchiesta di Danilo Dolci condotta in Sicilia intervistando contadini, operai, pescatori, donne e bambini (Dolci, 2008). Anche per Gavino Ledda, cresciuto nell'assolata e solitaria campagna sarda, il padre è un padrone, come racconta nel suo romanzo autobiografico. Costretto ad abbandonare la scuola a sei anni per intraprendere il mestiere di pastore, relegato anche per settimane in impervi e isolati posti di montagna a custodire il gregge, Ledda denuncia un'infanzia contadina drammatica, segnata da una violenza paterna che non gli risparmia percosse e solitudine. Un mondo contadino patriarcale sul piano della struttura familiare e della gestione dei rapporti, da cui il protagonista riesce a riscattarsi attraverso lo studio ostinatamente perseguito nonostante l'ostilità paterna (Ledda, 1977).

Siamo di fronte, quindi, a storie di vita radicalmente in contrasto con quelle idee pedagogiche, già ampiamente diffuse, almeno in apparenza, nelle società occidentali, dalle quali emerge la permanenza nel tempo, a dispetto dei mutamenti culturali che caratterizzano il susseguirsi delle generazioni, di forme di mentalità arcaiche che persistono, in modo più o meno latente, nelle mentalità collettive e nelle rappresentazioni individuali: concezioni e pratiche educative arcaiche che, se in certi periodi storici possono sembrare superate, scalzate dai mutamenti culturali emergenti, a volte sembrano riemergere dalla cenere, inaspettatamente, in tutta la loro drammaticità. 


\section{Riferimenti bibliografici}

Ariès Ph. (1973). L'enfant et la vie familiale sous l'ancien regime. Paris: Seuil (trad. it.: Padri e figli nell'Europa medievale e moderna. Roma-Bari: Laterza, 1983).

Becchi E., Julia D., a cura di (1996). Storia dell'infanzia. Roma-Bari: Laterza.

Becchi E., a cura di (1979). Il bambino sociale. Privatizzazione e deprivatizzazione dell'infanzia. Milano: Feltrinelli.

Bergman I. (1997). Lanterna magica. L'autobiografia del maestro del cinema. Milano: Garzanti.

Boas G. (1966). The cult of childhood. London: Warburg Institute, University of London (trad. it.: Il culto della fanciullezza. Firenze: La Nuova Italia, 1973).

Borruso F., Cantatore L., Covato C., a cura di (2014). L'educazione sentimentale. Vita e norme nelle pedagogie narrate. Milano: Guerini scientifica.

Braudel F. (1986). Une leçon d'histoire. Paris: Les Editions Arthaud (trad. it.: Una lezione di storia. Torino: Einaudi, 1988).

Cambi F., Ulivieri S., a cura di (1988). Storia dell'infanzia nell'Italia liberale. Firenze: La Nuova Italia.

Cambi F., Ulivieri S., a cura di (1990). Infanzia e violenza. Forme, terapie, interpretazioni. Firenze: La Nuova Italia.

Cantatore L. (2015). Parva sed apta mihi: studi sul paesaggio domestico nella letteratura per l'infanzia del $19^{\circ}$ secolo. Pisa: ETS.

Cavarero A. (2001). Tu che mi guardi, tu che mi racconti: filosofia della narrazione. Milano: Feltrinelli.

Covato C. (2007). Memorie discordanti. Identità e differenze nella storia dell'educazione. Milano: Unicopli.

Covato C. (2014). Idoli di bontà. Il genere come norma nella storia dell'educazione. Milano: Unicopli.

Covato C., Ulivieri S., a cura di (2010). Itinerari nella storia dell'infanzia: bambine e bambini, modelli pedagogici e stili educativi. Milano: Unicopli.

DeMause L., a cura di (1994). Storia dell'infanzia. Milano: Emme.

Dolci D. (2008). Racconti siciliani. Palermo: Sellerio.

Engels F. (1972). La situazione della classe operaia in Inghilterra, in base a osservazioni dirette e a fonti autentiche. Roma: Editori Riuniti.

Filograsso I. (2012). Bambini in trappola. Pedagogia nera e letteratura per l'infanzia. Milano: FrancoAngeli.

Foucault M. (1971). L'ordre du discours. Paris: Gallimard (trad. it.: L'ordine del discorso. Torino: Einaudi, 1972).

Foucault M. (1975). Surveiller et punir: naissance de la prison. Gallimard: Paris (trad. it.: Sorvegliare e punire. Nascita della prigione. Torino: Einaudi, 1977).

Freud S. (2003). Tre saggi sulla teoria sessuale. Torino: Bollati Boringhieri.

Gelmini A., a cura di (1892). Perez, Bernard, in Dizionario illustrato pedagogico. Milano: Vallardi.

Guarnieri P. (2006). Un piccolo essere perverso. Il bambino nella cultura scientifica italiana tra Otto e Novecento. Contemporanea. Rivista di storia dell'800 e del '900, 2: 253-284. 
Kafka F. (2011). Lettera al padre. Torino: Einaudi.

Ledda G. (1977). Padre padrone: l'educazione di un pastore. Milano: Feltrinelli.

Lombroso C., Marro A. (1883). I germi della pazzia morale e del delitto nei fanciulli. Archivio di psichiatria, scienze penali ed antropologia criminale, 4: 7-17.

Luatti L. (2016). Adulti si nasceva. Immagini e metafore letterarie sull'emigrazione minorile girovaga e di lavoro dall'Ottocento ai giorni nostri. Isernia: Cosmo Iannone.

Mantegazza P. (1889). La fisiologia dell'odio. Milano: Treves.

Miller A. (1987). La persecuzione del bambino. Le radici della violenza. Torino: Bollati Boringhieri.

Perez B. (1887). La psicologia dell'infanzia dai tre ai sette anni. Prefazione e note di C. Lombroso. Milano: Trevisini.

Pironi T. (2010). Da Ellen Key a Maria Montessori: la progettazione di nuovi spazi educativi per l'infanzia. Ricerche di Pedagogia e didattica, 1: 1-15.

Pollock L. (1983). Forgotten children: parents child relations from 1500 to 1900. Cambridge: Cambridge University Press.

Revelli N. (1977). Il mondo dei vinti. Testimonianze di vita contadina. Torino: Einaudi.

Riva M.G. (1993). L'abuso educativo: teoria del trauma e pedagogia. Milano: Unicopli.

Rousseau J.J. (1997). L'Emilio o dell'educazione. Milano: Mondadori.

Todorov T. (2002). Una fragile felicità: saggio su Rousseau. Milano: Se.

Trisciuzzi L., Cambi F., a cura di (1989). L'infanzia nella società moderna. Dalla scoperta alla scomparsa. Roma: Editori Riuniti.

Ulivieri S., a cura di (2014). Corpi violati. Condizionamenti educativi e violenze di genere. Milano: FrancoAngeli.

Vegetti Finzi S. (2015). Una bambina senza stella. Milano: Rizzoli.

Vovelle M. (1982). Idéologies et mentalités. Paris: F. Maspero (trad. it.: Ideologie e mentalità. Napoli: Guida, 1989).

Vidoni G. (1924). La delinquenza dei minorenni. Scaturigini e rimedi. Roma: Leonardo da Vinci.

Zoja L. (2000). Il gesto di Ettore. Preistoria, storia e attualità della scomparsa del padre. Torino: Bollati Boringhieri. 